\title{
Inclusion in an international training program for early-career engineers and scientists in the Thirty Meter Telescope partnership
}

McConnell, Nicholas, Barnes, Austin, Skidmore, Warren, Kupke, Renate, Lu, Jessica, et al.

Nicholas J. McConnell, Austin Barnes, Warren Skidmore, Renate Kupke, Jessica Lu, Scott Roberts, Ravinder Bhatia, Sandra Dawson, Gary H. Sanders, Lisa Hunter, "Inclusion in an international training program for earlycareer engineers and scientists in the Thirty Meter Telescope partnership," Proc. SPIE 11449, Observatory Operations: Strategies, Processes, and Systems VIII, 1144910 (13 December 2020); doi: 10.1117/12.2575654 


\title{
Inclusion in an international training program for early-career engineers and scientists in the Thirty Meter Telescope partnership
} Nicholas J. McConnell *a,b, Austin Barnes ${ }^{\mathrm{a}, \mathrm{c}}$, Warren Skidmore ${ }^{\mathrm{d}}$, Renate Kupke ${ }^{\mathrm{e}}$, Jessica Lu ${ }^{\mathrm{f}}$, Scott Roberts $^{\mathrm{g}}$, Ravinder Bhatia ${ }^{\mathrm{d}}$, Sandra Dawson ${ }^{\mathrm{d}}$, Gary H. Sanders ${ }^{\mathrm{d}}$, Lisa Hunter ${ }^{\wedge a}$

anstitute for Scientist \& Engineer Educators, University of California, Santa Cruz, 1156 High St., Santa Cruz, CA, USA 95064; ${ }^{b}$ Dept. of Physics \& Astronomy, California State University Sacramento, 6000 J St., Sacramento, CA, USA 95819; 'Scripps Institution of Oceanography, University of California, San Diego, 9500 Gilman Dr., La Jolla, CA, USA 92093; ${ }^{\text {Thirty Meter }}$ Telescope International Observatory, $100 \mathrm{~W}$ Walnut St., Pasadena, CA, USA 91124; 'University of California Observatories, 1156 High St., Santa Cruz, CA, USA 95064; 'Department of Astronomy, University of California, Berkeley, 501 Campbell Hall, Berkeley, CA, USA 94720; ${ }^{\text {Herzberg }}$ Astronomy \& Astrophysics, National Research Council of Canada, 5071 W Saanich Rd., Victoria, Canada, V9E 2E7

\begin{abstract}
The TMT Early-Career Initiative (TECI) is an innovative, evolving program designed to support inclusion in the Thirty Meter Telescope (TMT) International Observatory (TIO) by engaging graduate students and postdocs in TIO projects, and preparing them with skills required to contribute to the project and advance in their careers. TECI has an annual cycle that begins with a workshop that includes project management, instrument design, and teamwork sessions, and engages participants in projects that could lead to visits and new collaborations. Project teams are led by the participants themselves, who consult with a member of the relevant technical team or project staff. In this paper we describe the components of TECI, our approach to designing it, and outcomes from our early piloting in 2016-17, as well as our first full program in 2018-19.
\end{abstract}

Keywords: Times Roman, image area, acronyms, references

\section{TECI BACKGROUND}

The Thirty Meter Telescope (TMT) is one of a new class of extremely large telescopes that will allow us to see deeper into space and observe cosmic objects with unprecedented sensitivity. With its $30 \mathrm{~m}$ prime mirror diameter, TMT will be three times as wide, with nine times more area, than the largest currently existing visible-light telescope in the world. This will provide unparalleled resolution, with TMT images more than 12 times sharper than those from the Hubble Space Telescope. When operational, TMT will observe in wavelengths ranging from the ultraviolet to the mid-infrared, and will allow astronomers to address fundamental questions in astronomy ranging from understanding star and planet formation, to unraveling the history of galaxies and the development of large-scale structure in the Universe.

To be successful, the TIO partnership will need to work effectively together over the coming decades, and by first light, partners will need to be fully integrated into all aspects of TIO and have a talent pool positioned to maximize the scientific output of TIO. The geographic dispersion of the TIO partnership is vast, and challenges imposed by distance and cultural differences are notable even amongst those who study "team science". In addition, TIO's precursor, TMT Observatory Corporation, was incorporated solely as a U.S. partnership in 2007, and it was not until 2014 that these U.S. partners joined with the non-U.S. partners - Japan, India, China, and Canada — to form TIO. This has led to unbalanced participation in the partnership. For example, even though Japan is the largest contributor to TIO, the distribution of authors of the 2015 TMT Detailed Science Case shows that Japan, China, India and Canada each have far fewer authors (69 combined) than the U.S. (75 authors). Because disparities in the involvement of the partners could have far reaching effects, and will take years to ameliorate, TIO identified the inclusion of the full partnership as a high priority to be addressed as early as possible.

\footnotetext{
*n.j.mcconnell@gmail.com_^lunter@ucsc.edu
} 
In 2014, TIO initiated a collaboration with the Institute for Scientist \& Engineer Educators (ISEE) at University of California at Santa Cruz and University of California Observatories to design a new international training program. ISEE worked with the TIO community to develop, and now runs, the Thirty Meter Telescope Early-Career Initiative (TECI) to support early-career engagement in TIO, for all of the countries and institutions in the partnership. The goals of TECI are to:

1. Advance early-career scientists' and engineers' involvement with TMT

2. Contribute to making TMT teams more reflective of partner demographics

3. Support collaboration in the evolving TMT and US Extremely Large Telescope Program (US-ELT) partnership

Working from these three goals, we established outcome metrics (see Appendix), and launched an iterative design process to engage the TIO community in creating a program that would be inclusive of the diverse needs, interests, and backgrounds of the institutions and individuals that make up the partnership (Section 2). The resulting structure for TECI - implemented fully in 2018-2019 - includes a weeklong workshop and opportunities to contribute remotely or in-person to TMT and US-ELT projects (Section 3). Throughout the development of TECI we have aimed to support equitable participation from across the TMT partnership (Section 4.1). Finally, we report how TECI has evolved to more effectively support collaboration within the TMT partnership and has delivered valuable career development for its participants (Section 4.2).

\section{APPROACH TO TECI}

There were three major influences on our design: 1) the body of knowledge on effective professional development; 2) prior experience in designing, implementing and evaluating programs; and 3) input from the TMT community related to needs, constraints, and opportunities. Each are described in more detail below.

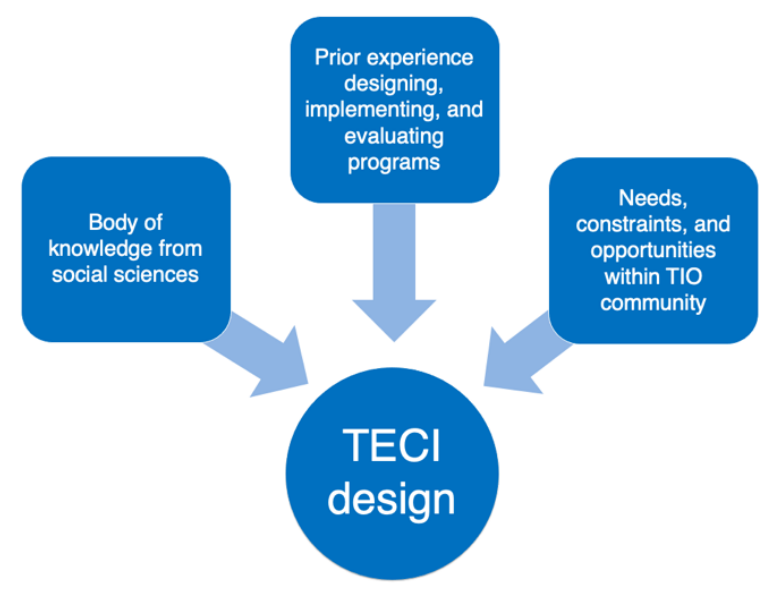

Figure 1. Major influences on the design of TECI.

\subsection{Using the social sciences to inform the design of TECI}

The body of knowledge established by the social sciences that can inform the design of programs like TECI is vast and comes from a range of disciplines. Psychologists have learned about training and development, such as the scientific principles that make training in teamwork and collaboration effective ${ }^{2}$. Researchers in the field of education have amassed a wealth of knowledge about teaching and learning, which has been made highly accessible through books that emphasize the practical implications of research findings ${ }^{3}$. There is also a great deal known from studying teacher professional development ${ }^{4-6}$. A theme that emerges from all of these fields of study is the importance of putting into practice skills that are learned, with coaching, feedback, and reflection. That is, listening to lectures is unlikely to do more than raise awareness, and the assumption that people will implement newly acquired skills later, on their own, is not well founded. 
Because a major goal of TECI is to promote inclusion across the TIO partnership, which includes people from a wide range of social and cultural backgrounds, the design of TECI was also informed by emerging research on diversity and inclusion, as well intercultural collaboration. For example, Shore et al. have advanced a definition of inclusion that includes satisfying individuals needs for both belongingess and uniqueness ${ }^{7}$, which ISEE uses to frame a range of professional development activities, and which was used to shape TECI activities. There are areas of overlap between inclusion and intercultural collaboration, but there is also a growing body of knowledge that was used as we developed TECI. For example, Osland \& Bird highlight the risk of culture-specific knowledge perpetuating stereotypes that become barriers ${ }^{8}$ and recommend:

- Being willing to question what is taken as "normal" according to one's previous knowledge and experience

- Ability to decenter one's perspective and take other perspectives in addition to one's own

- Cognitive flexibility: Adapt one's way of thinking according to situation or context

All of these areas of research informed how TECI was designed, along with practical experience and an analysis of needs, which are each described below.

\subsection{Using prior experience in designing, implementing, and evaluating programs}

The second major influence on the design of TECI was practical experience gained from designing, implementing, and evaluating programs with goals similar to TECI. The Institute for Scientist \& Engineer Educators (ISEE) partnered with the TIO Project Office and was tasked to work with the TIO community to design a program that could evolve over time as TIO moved into construction and then to operations. ISEE, a legacy of the NSF Center for Adaptive Optics (CfAO), has significant experience in designing and implementing programs $\mathrm{s}^{9,10}$ and using social sciences to inform the design and evaluation of programs ${ }^{11}$, as well as leading scientific and technical communities in the development of successful education and workforce development initiatives ${ }^{12}$. Lessons learned from this practical experience ranged in scope, from the process used in designing TECI, to strategies for recruiting participants, to pedagogical approaches to designing effective workshops, short courses ${ }^{13}$ and summer schools. A few examples include:

- Strategic engagement of stakeholders in the design process: For TECI to be successful the program would need to be shaped by the community it would serve. The experience gained from establishing CfAO's educational initiatives yielded many lessons that could be applied to working with the TMT community to design TECI. For example, after an initial needs analysis, gather momentum by proposing an initial program idea that is familiar, but not overly defined (e.g. an internship), which can be revised and built up as people can respond to it. The key to this strategy is continually balancing an openness to input with the need to make efficient progress in designing the program. We used this process throughout the development of TECI.

- Matching people and projects: Though many details needed to be figured out, we knew that the overarching goal for TECI was to get early-career participants engaged with TMT projects. A major lesson learned from previous ISEE programs such as the Akamai Workforce Initiative ${ }^{10}$ is the importance of not just having meaningful projects for students to engage in, but the careful matching of people to those projects. A good match will enable what is called in Akamai, a "productive project" - one that will be a valuable learning experience for the student and will be a valuable contribution to the mentor/host organization. Applying this experience to TECI meant that we would need to create ways for early-career people from across the TMT partnership to be matched with real TMT projects.

- Cycles of experiential learning, practice and reflection: All of ISEE's programs have prioritized learning followed by practice, and then reflection on what was learned and how to apply it. For example, central to the design of ISEE's Professional Development Program, participants learn about effective teaching, have a practical teaching experience, and then reflect on that experience ${ }^{14}$. This aligns with research, and differs from training efforts in which participants learn, but don't get to apply and reflect. In considering TECI, we prioritized this learn-practice-reflect cycle as a design principle.

- Recruiting a diverse pool of participants: Diversity, equity and inclusion are core values in all of ISEE's programs, which includes a commitment to recruiting participants from underrepresented, under-served, and marginalized communities. For example, ISEE's Akamai Program has been successful in advancing Hawai'i college students from underrepresented groups ${ }^{15}$, which begins with recruiting participants such as Native 
Hawaiians so that the program reflects the demographics of the community ( $25 \%$ Native Hawaiian). Strategies employed by Akamai and other ISEE programs are directly applicable to TECI so that women, individuals at smaller institutions in the TMT partnership, and other underrepresented groups could be included.

\subsection{Needs, constraints, and opportunities within the TIO community}

Informed by our prior experience, we were strategic in our design process for TECI. Through a series of meetings at TMT partner sites, we learned about the needs, interests, and constraints of the TMT stakeholders, which our design would need to address. We started with the idea of cross-partnership visits (alternatively called "exchanges" or "internships"), providing just enough structure for people we met with to be able to respond to. We asked people what would work, what would be gained, what the barriers would be, and what might get in the way of achieving the goals. We talked to graduate students, postdoctoral researchers, advisors, and project leaders from across the TMT partnership. As we learned from the community we continually revised and added detail to the design. In this early phase of our design work, we learned that TMT partners were enthusiastic about early-career participants (graduate students and postdocs) spending time at TMT partner sites and viewed it as an opportunity that would advance career development. Members of the community also saw it as a way to start new collaborations and build cohesion amongst the partners. However, there were a number of issues brought up that made it clear that simply funding visits was unlikely to have a significant impact on TMT and TMT partners, and which influenced the design of TECI. Some examples of things we learned early in our design process include:

- Extended visits for some, plus a smaller step for more: Extended visits will serve a very small group, and there needs to be components to the program open to a broader range of people. Also, an extended visit is a big leap that many students may not be willing to do without a smaller step first.

- Maximizing benefits from costly visits: Concerns were raised about the potential for slow start up times for visitors, as well as the potential for visitors to end up spending most of their time alone in an office.

- Targeted training in instrumentation: Training in instrumentation was identified by many partners as an area of important need for the future workforce.

- Professional skills with opportunities for practice: Professional skills, including project management, communication, cross-cultural and interdisciplinary collaboration, and teamwork were identified as skill sets broadly needed and often not gained in traditional programs.

- Cultural and language differences: TMT partners include individuals from different cultures, and who speak different languages. The program would have to address culture and language throughout all program components, including application and selection processes, preparation for working on a TMT project, virtual collaboration, and maximizing the outcomes of visits to TMT sites.

Using what we learned from the TMT community, together with our own experience, and informed by research, we came up with a preliminary design for TECI, and piloted components of that design in 2016. Participants in the 2016 TECI workshop participated in the first versions of our workshop sessions, and also spent part of the workshop contributing to the design of the future TECI program. In 2017 we offered a second workshop, which also included involvement of participants in feedback and design. In 2018 we offered the first version of TECI which we considered the full model of the program. We considered this version a success and planned to offer it again in 2019 in Hawai' $i$, which was postponed to 2020, and then eventually cancelled due to the COVID-19 pandemic. Future versions of TECI will use the 2018 design, with minor modifications and adaptations to align with the needs of the US-ELT program.

\section{COMPONENTS OF TECI}

The current structure of TECI includes two major components, developed through the approach outlined above. TECI comprises an intensive in-person workshop for all participants, and a suite of project experiences (TECI projects) that a subset of participants pursue in remote teams. TECI project teams may apply for a travel award to continue their work in person at a TMT or US-ELT partner site. Below we describe the TECI Workshop and TECI Project components in turn. 


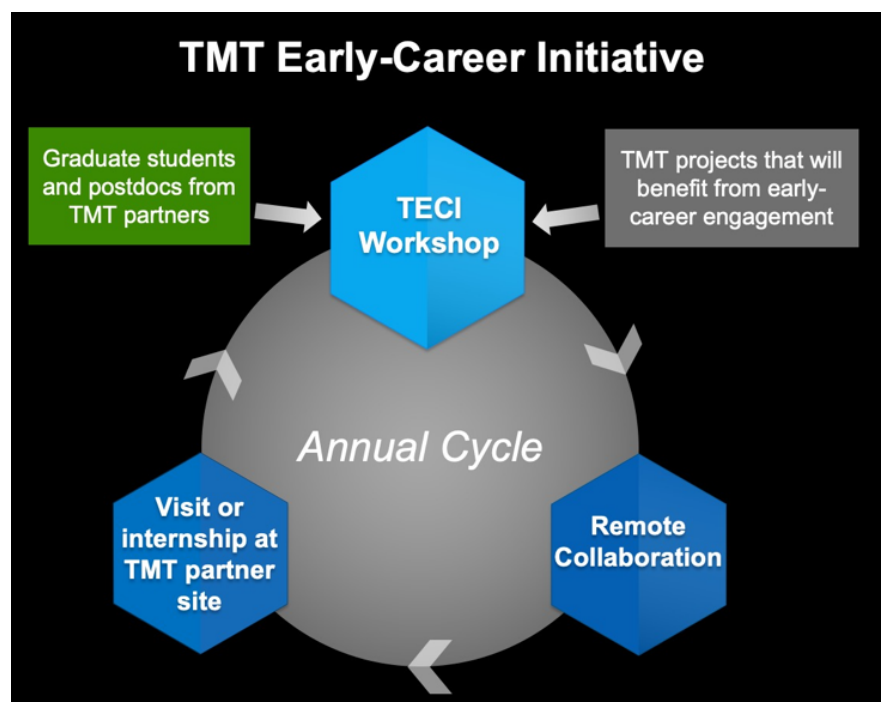

Figure 2. Annual program cycle for TECI. All participants come together for the TECI Workshop, and participants may continue the cycle with remote collaboration and an in-person visit to continue work on a TECI project.

\subsection{TECI Workshop}

The annual TECI Workshop brings the full TECI cohort together to learn new skills, gain initial engagement in TMT projects, and interact with the broader TMT community. TECI has held three workshops through 2020:

- 5-day pilot workshop in December 2016, Hilo HI, USA (35 participants)

- 7-day workshop in August 2017, Santa Cruz, CA, USA (42 participants)

- 7-day workshop in December 2018, Pasadena, CA, USA (49 participants)

The TECI workshops have hosted 111 participants in total, with some participants returning for multiple workshops. Additionally, we organized a 6-day workshop to be held in May 2020 in Victoria, BC, Canada. The 2020 workshop was canceled due to COVID-19, after we had accepted 49 participants.

During interactive sessions and group projects, workshop participants learn and practice skills such as working effectively on interdisciplinary and international teams, defining technical requirements based on science goals, and managing projects. Groupings for discussions and team-based activities are orchestrated so that participants interact with many of their colleagues from other countries, and work in mixed-gender teams. The workshop curriculum is organized by "strands:" major thematic elements that each include a series of activities designed to meet specific learning outcomes. Below we describe three strands in turn: instrument design; teamwork, collaboration and leadership; and project management. TECI Projects are described in Section 3.2, and their overlap with the TECI Workshop is a fourth curriculum strand.

\subsubsection{Instrument design}

The participants in TECI come from varied scientific and technical backgrounds, including theoretical and observational astronomy, astronomical instrumentation, and pure engineering disciplines. The instrument design component of TECI is structured to demonstrate the value of this broad range of backgrounds in the development of TMT instrumentation. It also serves as an introduction to the process of astronomical instrument design and systems engineering.

The strand includes a brief overview of types of astronomical instrumentation, and a description of TMTs first-light instrumentation already in design phase. Teams of 6-10 participants are then tasked with developing a compelling science case for a new TMT instrument, followed by deriving instrument requirements based on that science, advancing a technical conceptual design, and preparing and participating in a design review presentation. 
Each team is designed to have a variety of science and technical backgrounds, and participants are encouraged to work to their strengths as team members. The astronomers are tasked with flowing down instrument requirements from the science they wish to accomplish (i.e. spectral and spatial resolution, field of view, adaptive optics requirements) and those with more technical backgrounds contribute their expertise in instrument design.

Several TECI participants are chosen to form a Science Advisory Committee (SAC) whose role is to evaluate the science cases, technical risks and costs of each of the proposed instruments and rank the proposals in order of priority for further development. The SAC members are chosen to represent all TMT partners and a variety of astronomy research interests. The prioritization process exposes the SAC members and the instrument teams to the difficult decision-making process that large resource-limited projects must undertake.

In addition to a visit to the Caltech Optical Observatories instrument development labs, the 2018 workshop included a visit to TIO headquarters in Pasadena, where instrument team members gained access to TMT professionals for advice and guidance on specific aspects of their instrument designs. This activity allowed participants to build connections with experts within the TMT project.

\subsubsection{Teamwork, collaboration, and leadership}

One of the primary drivers behind the TECI Workshop has been to expose early-career scientists and engineers to the types of teamwork, collaboration, and leadership that are necessary for large, international and multidisciplinary projects like TMT. Through our conversations with TMT collaborators, feedback on early workshop pilot sessions, and ISEE's experience with building, facilitating, and maximizing efficacy of teams, we identified that workshop participants wanted and needed to prepare for professional careers in which they will work on international teams of various sizes comprised of individuals from different backgrounds, particularly emphasizing crosstalk between engineers and scientists on technical projects requiring both. The teamwork, collaboration, and leadership strand is designed to be an interwoven thread supporting the other workshop activities described in this section, and includes small group discussions early in the workshop, another discussion later in the workshop to reflect on the process and what effective international collaboration does and should look like, and a final moderated panel of scientific and technical leadership who work extensively in international and interdisciplinary teams.

TECI Workshop participants first think about and discuss in staff-facilitated discussions what it means to work across cultures, language barriers, time zones, geographical locations, as well as technical disciplines. They consider how teams can anticipate and overcome the associated challenges, and what individuals as formal or informal leaders and team members can do to help achieve effective collaboration. Participants then put these ideas into practice through the instrument design, project management, and TECI projects, reflect on the process individually and in small group discussions, and revise their ideas together as they prepare to leave the workshop and begin working on these kinds of teams on the TECI Projects or other professional projects. The final panel of international scientific and technical leaders gives participants the chance to engage directly with and ask questions of individuals from all over the world who have decades of collective international collaborative experience.

The TECI Workshop includes an additional leadership strand experience for returning, multi-year participants or others more advanced in their careers. These participants volunteer for leadership roles in workshop components, and as part of their preparation the workshop includes small group discussions focused on specific strategies used by leaders of international and interdisciplinary teams.

\subsubsection{Project management}

The project management strand consists of a structured, interactive activity over one full afternoon of the workshop. The activity uses a project management scenario developed by Dave Palmer - upgrading a camera for a small observatory and tasks teams of 3-4 participants to plan the project timeline, allocate observatory staff time, and create a rudimentary Gantt chart. There is then an execution phase wherein each team is given two scenarios (for instance, a key staff member is reassigned to a different project) and must adjust their plan in response. This team exercise is bookended by roundtable discussions about project planning and assessing risks. Our goals for the project management strand are achieved if by the end of the activity, participants can demonstrate that their project plan:

- Defines tasks that accomplish the project requirements in a verifiable manner 
- Indicates the owners and duration of each task, and dependencies between tasks

- Indicates which tasks define the project's critical path, and which can float

- Considers time and budget risk, and incorporates contingency

In 2018 the project management activity occurred on the first day of the TECI Workshop and acted as an immediate stimulus for participants to work with people from other institutions and countries. Later in the workshop, the instrument design activities and TECI projects provided opportunities to reinforce and apply project management concepts and tools.

In addition to workshop activities that compose the strands above, the TECI Workshop includes presentations on the status of TMT instruments and overall TMT project, and tours of local facilities where telescope or instrument components are being developed. In order to help participants build connections with each other and with members of the TMT project, the workshop includes a poster session where participants can showcase the work they are pursuing at their home institution.

\subsection{TECI Projects}

The Projects component of TECI was developed to enable deeper engagement between TECI participants and TMT project members and to increase international collaboration among early-career engineers and scientists beyond the setting of the TECI Workshop. This component was designed to match people to projects, and maximize the benefit of any resourceintensive travel, which we identified as potential issues in our early design process (Section 2.2 and 2.3). Although only a fraction of TECI projects culminate with in-person travel, we have created infrastructure to ensure these visits are purposeful, and are accessible to a large swath of the TECI cohort, via a proposal stage.

TECI works with TMT science, instrument, and operations teams to identify technical projects that will benefit from engaging early-career people. Our aim for project scope is something that engages a team of participants for a few hours per week, extended over $\sim 2-6$ months ( $\sim 100$ person hours). Some projects may engage a small team for a few weeks of concentrated effort. Following a project introduction session at the TECI Workshop, an initial team of participants is assigned to each project, based on participant preferences and our requirement that each project team must be multinational. Each project has a consultant from the relevant TMT team, who is available (at the Workshop or remotely) to clarify background information and expected deliverables.

Table 1. Criteria for remote continuation of TECI projects

\begin{tabular}{|l|}
\hline \multicolumn{1}{|c|}{ Criteria for remote continuation of TECI projects } \\
Benefits to participants \\
1. Develops participants' international collaboration skills \\
2. Supports participants' work at their home institutions \\
3. Makes use the expertise and strengths of each individual team member \\
4. Strengthens connections between participants and TMT project \\
Benefits to TIO \\
5. Enhances international collaborations, especially within the partnership \\
6. Creates a finite product of value to TMT project \\
Merits of Project Approach \\
7. Strength of the proposed approach \\
8. Value added by team approach (as opposed to individual work) \\
9. Feasibility of project timeline \\
\hline
\end{tabular}

TECI project teams are assigned toward the beginning of the TECI Workshop and have several hours of working time throughout the week, wherein they establish team roles and project milestones, begin performing project tasks, showcase their preliminary work, and determine what next steps would be. Though participants work initially on assigned projects and teams, each individual may choose at the end of the workshop to: 
- Propose to continue remotely after the workshop, on a team with their current project

- Propose to continue remotely after the workshop with a different project team

- Stop working on any TECI project

Each project team that forms (or stays together) at the end of the TECI Workshop submits a proposal describing the remaining project milestones and deliverables, a proposed approach and timeline for working remotely with a TMT consultant, and how much time each project member can commit. Each proposal is evaluated by TECI staff as well as any person who would serve as a consultant for the proposed project. In Table 1 we list our evaluation criteria, which we share with participants in addition to a proposal template. Beyond these criteria, proposals to continue remotely are approved if a consultant agrees to work with the team during their specified timeline.

In a separate proposal step, project teams or individuals may propose to visit a TMT site in order to collaborate with individuals there toward advancing the project. The duration can be a short visit ( 1-2 weeks), or longer and more like an internship, and depends upon the project, availability of consultants and participants, and costs. Typically a visit is proposed after a team has demonstrated progress working remotely. The proposal must outline how the project can be uniquely advanced through in-person work, and how participants will take advantage of their opportunity to visit another site and meet with people there, beyond working specifically on their TECI project.

Table 2. Summary of 2018-2019 TECI projects and their outcomes.

\begin{tabular}{|c|c|c|c|c|c|}
\hline $\begin{array}{l}\text { Project started during } 2018 \text { TECI } \\
\text { Workshop }\end{array}$ & $\begin{array}{c}\text { Completed } \\
\text { during } \\
\text { workshop }\end{array}$ & $\begin{array}{l}\text { Propose to } \\
\text { continue } \\
\text { remotely }\end{array}$ & $\begin{array}{c}\text { Remote } \\
\text { work } \\
\text { approved }\end{array}$ & $\begin{array}{l}\text { Travel } \\
\text { award }\end{array}$ & $\begin{array}{l}\text { SPIE } \\
\text { paper }\end{array}$ \\
\hline Web tool for IRIS astrometry budget & & $\checkmark$ & $\checkmark$ & $\checkmark$ & $\checkmark$ \\
\hline Pinhole mask test unit for NFIRAOS & & $\checkmark$ & $\checkmark$ & $\checkmark$ & $\checkmark$ \\
\hline $\begin{array}{l}\text { Use cases for NFIRAOS+IRIS PSF } \\
\text { reconstruction }\end{array}$ & & $\checkmark$ & $\checkmark$ & & \\
\hline TMT data archive use cases & & $\checkmark$ & $\checkmark$ & & \\
\hline Improving Detailed Science Case web tool & & $\checkmark$ & $\checkmark$ & & \\
\hline Finite element analysis for WFOS optics & $\checkmark$ & & & & \\
\hline WFOS observing use cases & & & & & \\
\hline Planetary Systems Imager optical relays & & $\checkmark$ & & & \\
\hline Optical camera behind NFIRAOS & & $\checkmark$ & & & \\
\hline
\end{tabular}

Our first full implementation of the TECI Projects component took place during and after the 2018 TECI Workshop. In Table 2 we summarize the projects that were sourced from the TMT community and how far each was advanced during TECI. Five teams, comprising 20 participants out of 49 who attended the 2018 TECI Workshop, continued working remotely on TECI projects. Two teams who made substantial progress working remotely received travel awards for an 
in-person visit in fall 2019, to culminate their project work and collaborate at a TIO partner site. Both teams have reported the outcomes of their project in SPIE proceedings ${ }^{16,17}$.

\section{EVALUATING TECI OUTCOMES}

To evaluate what TECI has achieved since our pilot workshop in December 2016, we have defined indicators of progress toward the program's three major goals, which are to:

- Advance early-career scientists' and engineers' involvement with TMT

- Contribute to making TMT teams more reflective of partner demographics

- Support collaboration in the evolving TMT and US Extremely Large Telescope Program (US-ELT) partnership

In Appendix A we list concrete long-term outcomes corresponding to each goal, as well as medium-range indicators that address the four-year timeframe TECI has been active so far. The medium-range indicators allow us to weigh the degree to which TECI is currently poised to advance TIO's long-range goals for early-career involvement, collaboration, and advancement. They also help frame how TECI should further develop in order to bridge from current indicators to longterm outcomes. For short-term evaluation, we also consider participants' response to the workshop through surveys and their perspective a year or more after participating (Figure 3). Our evaluation metrics and processes have been developed to include contributions to the US-ELT program in future TECI cycles.

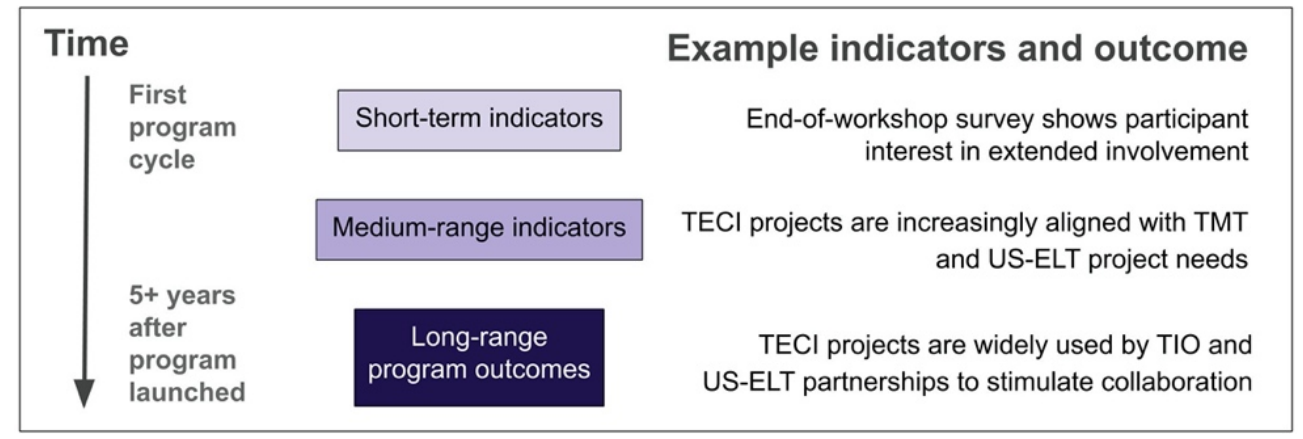

Figure 3. Example of short-term and medium-range indicators that convey progress toward a long-range outcome for TECI

Individuals who are able to participate in TECI represent a small fraction of the TMT project workforce, and in defining outcomes we consider the scope of collaboration or change in the TMT project that could reasonably be traced to TECI. In many cases our medium-range indicators follow the evolution of TECI toward a model that advances projects valued by TIO and US-ELT. Our expected long-term outcomes will guide how we monitor the advancement of previous TECI participants (TECI alums) in their own careers and in TIO and US-ELT roles.

\subsection{TECI Participation 2016-2019}

TECI has succeeded in bringing together workshop cohorts with strong representation from each TIO partner. Of 111 individuals who participated in TECI from 2016-2019, each TIO partner has sent at least 13. In Figure 4 we show the relative representation of TIO's partners, the U.S. community at large, and the non-U.S. community at large. For comparison to TECI, Figure 4 includes the relative fractions by community for contributors to the 2015 TMT Detailed Science Case ${ }^{18}$, and for members of TMT International Science Development Teams (ISDTs) as of $2019^{19}$. Unlike the Detailed Science Case and International Science Development Teams, TECI has not previously been open to individuals outside the TIO partnership, except under special circumstances. The relatively high fraction of TECI participants from the University of California (UC) can be attributed in part to the location of the 2017 and 2018 TECI Workshops, and to travel funding provided by UC Observatories.

We employed several strategies for recruiting and selecting TECI participants, that enabled us to build a multi-national cohort with sizable interest from each partner. First, we devoted substantial resources to in-person recruiting trips to each 
partner country, focusing on sites where several individuals or entire groups were actively involved in TMT. Although we also rely on word-of-mouth recruiting through colleagues in each country, in-person visits allow us to thoroughly convey what TECI is and what participants can gain, and to field questions. Following the 2016 and 2017 workshops, we made follow-up trips to countries and institutions who supplied fewer applicants than expected, to continue developing relationships and promoting the program. In both cases, our applicant numbers from the initially underrepresented partners increased the following cycle. Our second strategy was to open the program application and inform selected participants of acceptance several months in advance of the workshop dates, in order to accommodate long processing times for international travelers securing visas. Third, we considered each partner independently when we made selection decisions. This enabled us to select a relatively balanced cohort (in effect, holding a minimum number of spots for each partner) in spite of a lopsided distribution in applications: the corollary is that applicants from some partners faced a far more competitive selection process than from others.

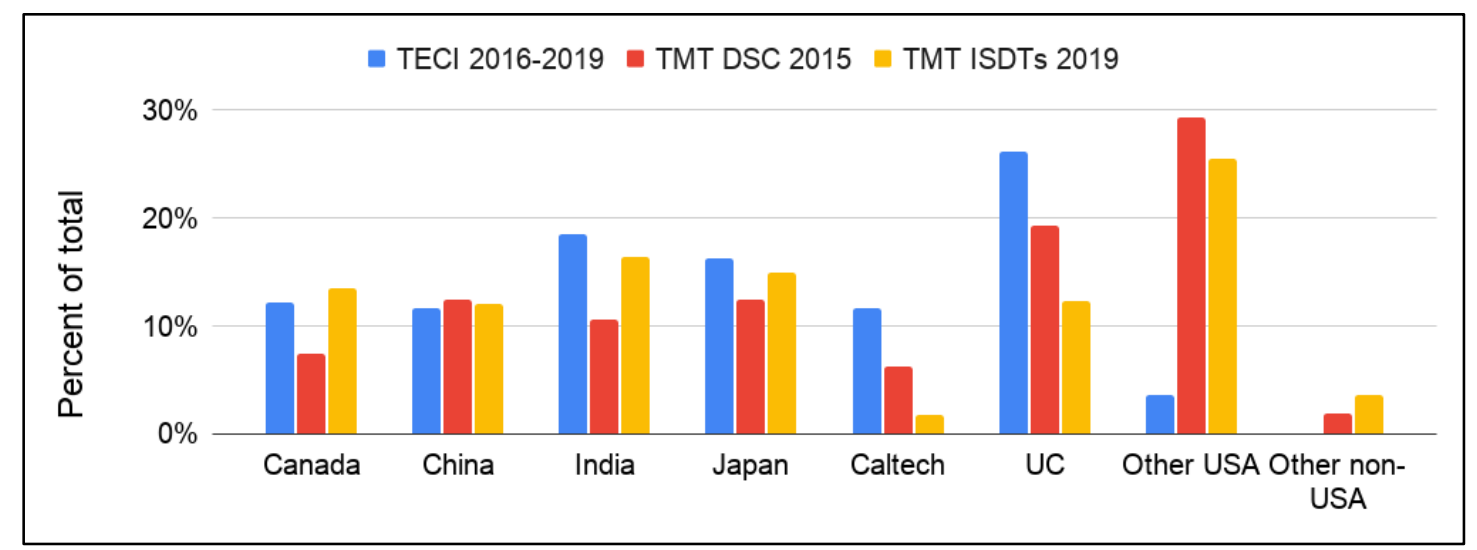

Figure 4. Relative participation of TIO partners, USA engineers/scientists outside the TIO partnership, and non-USA engineers/scientists outside the TIO partnership. Participation in TECI is compared to participation in the 2015 TMT DSC and TMT ISDTs as of 2019.

For the first time in 2019, TECI implemented the TECI Projects component with remote continuation after the 2018 TECI Workshop, and two project teams successfully proposing in-person visits. Our aim was to have a similarly balanced representation of TIO partners in the subset of participants ( 20 in total) who continued working on TECI projects. Whereas project teams assigned initially during the TECI Workshop are designed to be multi-national, participants were free to compose their own teams for continuing TECI project work. At least one person from each TIO partner continued working on a TECI project in 2019, and all of the continuing teams were multinational. In Figure 5 we compare the distribution by partner of TECI project continuers to the distribution for all participants in TECI from 2016-2019.

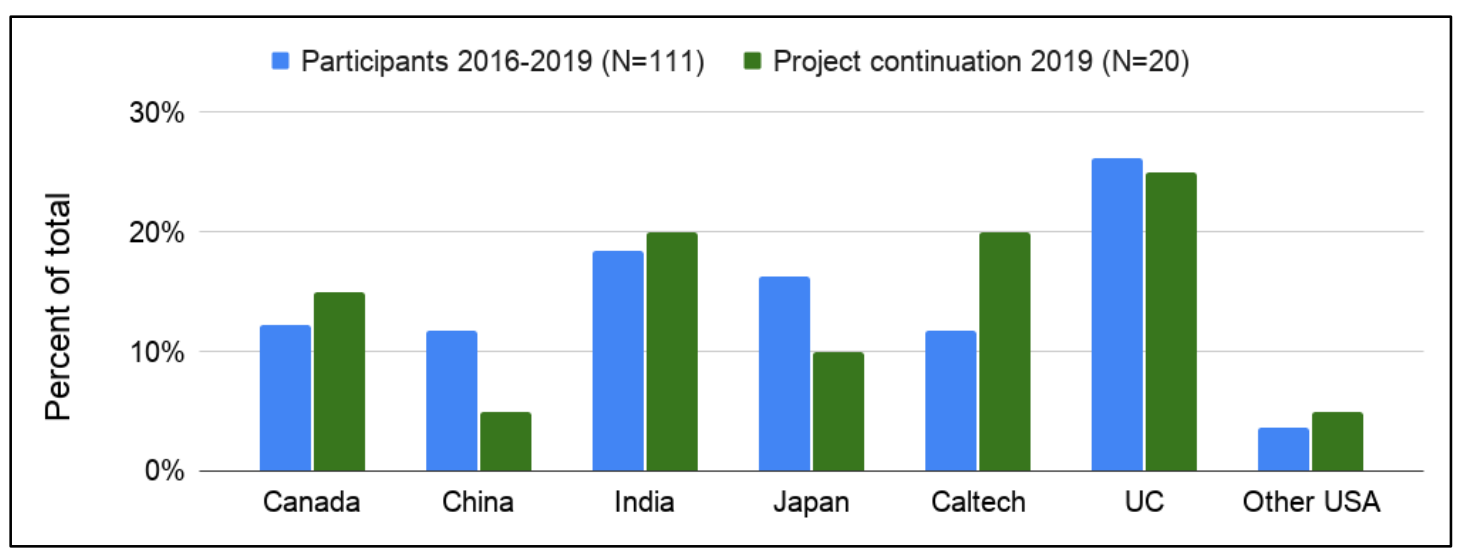

Figure 5. Relative participation of TIO partners and USA engineers/scientists outside the TIO partnership, for all TECI events from 2016-2019 and for TECI project continuation after the 2018 TECI Workshop. 
In addition to balancing partner representation, TECI shares with TIO the goal of increasing the representation of women at all levels and in leadership roles. Our TECI cohort from 2016-2019 comprises 63 men (57\%) and 48 women (43\%). As a point of comparison, the composition of TMT ISDTs in 2019 was $73 \%$ men and $27 \%$ women ${ }^{19}$. Although we have not created recruiting materials specifically targeting female engineers and scientists, we have upheld relationships with several advocates for gender equity in leadership roles throughout the partnership, who recognize the importance of and contribute to targeted word-of-mouth recruiting. In each program cycle we strive to select at least $50 \%$ women from each TIO partner with a sufficient number of qualified female applicants.

In addition to recruiting and selection, we strive to create an environment at the TECI Workshop that empowers all participants and disrupts unconscious patterns of bias $^{20}$. In addition to workshop sessions on inclusive teamwork practices (Section 3.1.2), there are many opportunities for participants to work in multinational, mixed-gender teams. Many of our workshop instructors have participated in other ISEE programs and received instruction in facilitating group dynamics. In TECI, instructors guide table discussions and team activities to help balance who contributes and whose ideas are considered. Finally, we strive for both gender and partner balance when assigning advanced roles during the instrument design sessions (team lead or SAC member - see Section 3.1.1). Through 2019, 12 men and 9 women have held an advanced role, and at least two individuals from each TIO partner. To the degree that our various interventions shape participants' experience, they support TECI's aims for effective professional and career development applied equitably. Our past participants (alums) are also important agents for word-of-mouth recruiting, which is strengthened when alums from many backgrounds report a positive experience.

\subsection{TECI Outcomes 2016-2020}

From TECI's 2016 pilot workshop through the 2018 workshop and 2019 TECI projects representing the full TECI program, we have adapted TECI to more effectively support major goals and long-term outcomes. In these first few years, the program has produced several indicators of progress, especially toward the goals of early-career engagement with TIO and supporting collaborations within the TIO and US-ELT partnership. Although several longer-term outcomes are not yet assessable, we highlight recent progress in TECI alums' involvement in projects and collaborations for TIO. For additional insight to TECI's impacts for early-career participants, we share alums' perspectives on how TECI has served their career interests and trajectories.

\subsubsection{Products and evolution of TECI}

For objective evidence of TECI's impact upon TMT collaborations and individual members, we consider products that have been generated through TECI projects, TECI alums' participation in TMT teams and events, and ways that TECI participants have been recognized for their contributions to TMT. We highlight how the Projects component of TECI has evolved to better enable project outcomes that are valuable to TIO and US-ELT.

TECI projects debuted in 2017, with a mix of use-case projects related to TMT science and operations goals (for instance, developing and compiling use cases for the eventual TMT data archive) and projects developing ideas for early-career engagement in TIO. Although the latter projects provided some input toward our further development of TECI, they were not paired with resources for further development (in the form of funding, or consultants' time following the 2017 TECI Workshop). While the use-case projects were an early model for work that would address immediate needs within TIO, the 2017 versions stalled due to the absence of program infrastructure to shepherd teams through a remote work phase.

Between the 2017 and 2018 TECI Workshops we persisted in meeting with members of first- and later-generation TMT instrument teams, who identified a handful of projects that would advance their work. Devising each project required iterative collaboration wherein we clarified TECI's goals and constraints and refined initial project ideas toward versions appropriate to TECI participants' expertise and commitment. At the 2018 Workshop we introduced new TECI projects connected to the NFIRAOS, IRIS, WFOS, and PSI instruments. Additionally, our full program model in 2018-19 included new infrastructure in the form of proposals (including guidelines and proposal templates distributed to participants), workbreakdown templates for project teams to use, and stronger coordination between TECI staff and TIO project consultants.

Our efforts yielded the suite of 2018-19 TECI projects listed in Table 2. The strongest outcomes are from two projects supporting TMT instrument teams, that each culminated in a weeklong visit, a design or software product handed over to a TMT instrument team, and an SPIE publication: 
- A team of three TECI participants from IIA (India), UCSD (USA), and GMT (USA: US-ELT) developed a webbased software tool to calculate the TMT astrometry error budget for different science cases, in collaboration with Jessica Lu (TMT IRIS science team) and Matthias Schöck (TIO). In September 2019 the team traveled to UC Berkeley for a one-week visit with Jessica Lu and Matthias Schöck, during which they refined the astrometry calculator and updated the web based tool. This project is reported by Ranka et al. ${ }^{17}$

- A team of three TECI participants from University of Victoria (Canada), NIAOT (China), and Caltech (USA) designed a new astrometric calibration unit to feed the NIRC2 AO instrument at the W. M. Keck Observatory, as a testbed for astrometric calibration for TMT NFIRAOS. This project is reported by Lin et al. ${ }^{16}$

The two projects above illustrate how the evolution of TECI projects has poised us to advance TIO collaborations, include early-career members from disparate institutions, and enable formal recognition for participants' contributions to TIO. As the scope of TMT's collaboration with the U.S. astronomy community has expanded, we have begun working to involve other branches of the US-ELT partnership in TECI Projects. In 2020, the National Science Foundation's National OpticalInfrared Astronomy Research Laboratory (NOIRLab) submitted a project with the Community Science \& Data Center, positioning TECI to expand to include the broader US astronomy community.

Beyond collaborations seeded at TECI, we aim to assess how TECI alums engage with TIO and US-ELT as they continue in their careers. A simple metric is the number of TECI alums who are members of teams developing TMT science and instrumentation. In 2018, seven instrument collaborations submitted white papers to the TMT Science Advisory Committee, presenting concepts for second-generation TMT instruments. Four TECI alums were co-authors for various white papers, representing four different instrument concepts. Separately as of 2019, eight TECI alums are participating on TMT ISDTs.

In addition to co-authorship and ISDT membership that can be supplied readily through the TMT Project Office, we expect that several TECI alums are contributing to TIO through projects at their home institution: in fact, their involvement often factors into their application to participate in TECI. At the time of this publication we have not comprehensively tracked the individual involvement of each alum, nor membership in the numerous TMT project teams and sub-teams. Our recent survey presented below offers some insight to how many alums are currently involved in TMT projects (13 of 55 respondents in July 2020). However, the survey response rate varies by TIO partner, as does the degree of partner involvement in TMT engineering projects - including cases where an individual's involvement in TIO precedes their participation in TECI. Responses collected from approximately half our cohort only provide a partial window to the impact TECI may be having on early-career engagement with TIO and US-ELT collaborations.

In coordination with the TMT Project Office, we have embraced the annual TMT Science Forum as another venue where TECI participants can expand their connections with the broader TMT community. Since TECI's inception, 41 TECI participants have attended the Science Forum in Mysuru, India (2017) or Pasadena, USA (2018). A 2017 Science Forum presentation featured a conceptual design for a fast-cadence spectrograph to perform transient follow-up observations (Fast SHOOTER), devised by participants in TECI's instrument design activity. This proved a timely contribution to the Forum's focus on developing second-generation instrument concepts for TMT, and illustrates the potential for TECI participants and activities to infuse ideas into the TIO and US-ELT collaborations. The 2018 TECI Workshop was held in Pasadena immediately before the 2018 Science Forum, enabling participants and senior project members to interact throughout the week.

\subsubsection{Participant perspectives}

Participant perspectives have been an important part of the ongoing design of TECI. In 2016 and 2017, participants engaged in workshop sessions intentionally designed to have participants actively involved in creating TECI. The 201819 version of TECI was the first full model, incorporating the feedback from prior year participants, but revisions will continue and TECI will adapt to the evolution of the TIO project. We collect participant perspectives at the end of each workshop, and will occasionally poll alumni. Self-report surveys and polls do not replace objective measures of outcomes $^{21}$, but they can provide feedback on program design, early indicators of outcomes, and often elicit testimonials that complement and add richness to other outcome metrics.

In end-of-workshop surveys from 2017 and 2018 (completed while participants were still convened in person), an overwhelming majority of participants $(86 \%)$ rated the workshop as "very valuable" ( 4 on a likert scale from 1- 
4). However, this rating only reflects the immediate impact of the TECI experience - at its most heightened impression and it does not offer any nuance toward the specific aspects of TECI that may prove valuable over time. In July 2020 we polled alums of the 2016, 2017, and 2018 TECI Workshops on their involvement in TMT projects and on the value of TECI toward their career development. We were able to contact 98 of 111 TECI alums, and 55 responded.

Our poll was designed to evaluate how TECI alums perceived the value of the program, in their own words. We were especially interested in identifying cases where TECI provided a unique opportunity relative to participants' formal training and daily work as early-career engineers and scientists. After asking alums to identify their current positions and involvement with TIO projects, we posed three optional free-response questions:

- Is there anything you want to share about how your experience with TECI has enabled you to work with TMT?

- Is there anything you want to share about how your experience with TECI has enabled you to pursue other work related to telescopes and astronomy?

- Any other comments you would like to share?

Although the free-response questions were optional, alums volunteered a broad range of comments and insights. We reviewed their responses and identified several themes, which we present in Table 3. Our determination of themes is informed by similarities in multiple alums' responses, as well as our own understanding of the experiences TECI was designed to provide. Once themes were identified, we re-read each alum's free responses and mapped them to the relevant themes. At the end of this process we combined a few initial themes with narrow distinctions into broader ones, obtaining

Table 3. Summary of themes from July 2020 survey of TECI alums. Example responses are taken directly from the survey.

\begin{tabular}{|l|l|}
\hline Valuable aspect of TECI & Example response \\
\hline $\begin{array}{l}\text { Opportunity to work on TMT } \\
\text { projects } \\
\text { (7 of 55, 13\%) }\end{array}$ & $\begin{array}{l}\text { TECI has definitely given me great opportunity early on to interact and connect } \\
\text { with a lot of the TMT team members and Scientists directly. This has been a } \\
\text { great help for me as it also allowed me also to travel to a different partner } \\
\text { country and be part of small projects. As a Junior postdoc or PhD student this } \\
\text { was very valuable to my career. }\end{array}$ \\
\hline $\begin{array}{l}\text { Networking opportunity, in } \\
\text { general } \\
\text { (14 of 55, 25\%, not including } \\
\text { row above) }\end{array}$ & $\begin{array}{l}\text { [TECI] has enabled me to get insights on how a organization like the TMT } \\
\text { works and what the people in the organization focus on. The workshop, I } \\
\text { attended, gave me the opportunity to meet people working in the TMT } \\
\text { consortium. }\end{array}$ \\
\hline $\begin{array}{l}\text { Opportunity to learn and } \\
\text { practice inclusive approaches } \\
\text { to international teamwork } \\
\text { (13 of 55, 24\%) }\end{array}$ & $\begin{array}{l}\text { Only TECI have given me the opportunity to closely work with other young } \\
\text { aspirants from different partner countries, which built confidence in me in terms } \\
\text { of communication, team work and work in different work coulture [sic]. }\end{array}$ \\
\hline $\begin{array}{l}\text { Exposure to new disciplines } \\
\text { (9 of 55, 16\%) }\end{array}$ & $\begin{array}{l}\text { [TECI] clearly broadened my view on managing big science project [sic] and } \\
\text { gave me a new perspective from the engineer point of view. }\end{array}$ \\
\hline $\begin{array}{l}\text { Training for systems } \\
\text { engineering or project } \\
\text { management skills used later } \\
\text { (9 of 55, 16\%) }\end{array}$ & $\begin{array}{l}\text { Participating in [TECI] helped me gain insight into the planning and execution } \\
\text { of TMT, which I can use in my current position that involves implementing and } \\
\text { designing large science programs/projects, and which I would not have gotten } \\
\text { elsewhere during my PhD work. }\end{array}$ \\
\hline $\begin{array}{l}\text { Training for instrumentation } \\
\text { skills used later } \\
\text { (5 of 55, 9\%) }\end{array}$ & $\begin{array}{l}\text { [TECI] was one of the first places that helped me understand the bridge } \\
\text { between astronomy science goals and telescope engineering. This helped me } \\
\text { later in writing proposals for other telescopes, in understanding telescope spec } \\
\text { documents when attempting to reduce data, and in learning about how [...] } \\
\text { engineering limitations interplay with astronomy science goals. }\end{array}$ \\
\hline
\end{tabular}


the list presented in Table 3. In Table 3 we also indicate the prevalence of each theme - effectively, where more or fewer alums recognized value in TECI. While the unconstrained nature of our questions allows for emergent expression of TECI's value to participants, we caution that this approach may not be an effective absolute metric of TECI's perceived or actual value (whereas the likert scale question following each workshop is a direct quantitative probe but very limited in scope).

Among the themes we identified, the largest fraction of poll respondents emphasized TECI's value in providing opportunities to meet and work with people from different countries, or with expertise that complemented their own disciplinary knowledge. Several alums also described TECI as a unique experience that supported their skill development, most frequently citing how it helped them conceptualize a large project at a high level and employ project management or systems engineering concepts. TECI may offer a novel window to these disciplinary areas, whereas the large majority of participants have degrees in astronomy, physics, optical engineering, mechanical engineering, or technology development. In a few cases, alums' TECI experience even stimulated them to consider a new career direction in project management or systems engineering.

Finally, we highlight one response that has not recurred as a theme so far, but nonetheless is strongly aligned with TECI's long-term goals and demonstrates the potential for TECI to have a major impact on participants' career advancement. One alum shared that meeting with mid-level and senior observatory staff during the TECI Workshop encouraged her to pursue an open position at an observatory, that she ultimately was hired for:

"My current project manager position at [a major observatory] is the result of my attendance of the [TECI Workshop] (through the enabled networking activities with other [project managers and systems engineers] working in the field of astronomy and instrumentation, that occurred during the workshop)."

As the TMT and US-ELT projects continue advancing, we expect many more opportunities to assess the involvement of early-career engineers and scientists who have been introduced or influenced by TECI. One insight we have gleaned from the preliminary evaluation reported here is the close intertwinement of our goals to support the development of collaborations - often through relatively small teams - and to advance individuals' involvement in the much larger TIO and US-ELT projects.

\section{CONCLUSIONS}

TECI is an evolving program designed to support inclusion in TIO by engaging graduate students and postdocs in TIO projects and preparing them with skills required to contribute to the project and advance in their careers. The program was designed using research from the social sciences, direct experience with similar programs, and input from the TIO community. We used an iterative design approach to build the program over time and which combined ongoing needs analysis and community input with piloting and revision. The full model of the program was offered in 2018-19 and began with a workshop that included project management and systems engineering, instrument design, and teamwork sessions, and engaged participants in projects that could lead to visits and new collaborations. Workshop sessions included preparation for working effectively and inclusively on multinational teams, with many opportunities to practice these skills during workshop sessions, such as working in a small group to come up with a conceptual design for an instrument. The workshop included sessions to engage participants in TIO projects, and some teams continued working on projects after the workshop, as an intended part of TECI's design.

Part of the TECI design process included identifying outcome metrics that spanned short, medium, and long-term indicators of success. Short-term outcome measures include the success in recruiting participant cohorts that represent the partnership and are close to balanced gender, and self-reports from participants about the value of TECI in their career advancement. One of the major goals of TECI is to engage participants in TIO, and the incorporation of real TIO projects into the workshop is the mechanism for initiating new collaborations. This aspect of the program model has been successfully demonstrated: two teams of TECI participants completed projects which are reported in SPIE papers. TECI has growing involvement from the TIO community and is now poised to support the TIO project as it evolves and moves toward first light. 


\subsection{Acknowledgments}

TECI has developed from the efforts of numerous individuals and teams. In particular, we thank Christophe Dumas, Fengchuan Liu, and Claire Max for ongoing support of TECI, as well as the TMT Workforce, Education, Public Outreach, and Communication (WEPOC) group, including Gordon Squires, Wako Aoki, Yiping Wang, Samir Dhurde, Eric Chisholm, Mitch Aiken, and Janesse Brewer. Rafael Palomino, Maureen Savage, Dave Palmer, and Hanne Buur have made substantial contributions to TECI's curriculum. TECI participant selection and the TECI Workshop would not be possible without the administrative and logistical support of Nicole Mattacola and Diana Bisel. Finally, we thank Tamara Ball for providing input on TECI outcomes and the preparation of this manuscript.

TECI was funded by the TIO Project Office in Pasadena, California and University of California Observatories.

The TMT Project gratefully acknowledges the support of the TMT collaborating institutions. They are the California Institute of Technology, the University of California, the National Astronomical Observatory of Japan, the National Astronomical Observatories of China and their consortium partners, the Department of Science and Technology of India and their supported institutes, and the National Research Council of Canada. This work was supported as well by the Gordon and Betty Moore Foundation, the Canada Foundation for Innovation, the Ontario Ministry of Research and Innovation, the Natural Sciences and Engineering Research Council of Canada, the British Columbia Knowledge Development Fund, the Association of Canadian Universities for Research in Astronomy (ACURA), the Association of Universities for Research in Astronomy (AURA), the U.S. National Science Foundation, the National Institutes of Natural Sciences of Japan, and the Department of Atomic Energy of India.

\section{REFERENCES}

[1] National Research Council. Enhancing the Effectiveness of Team Science. (Washington, DC: The National Academies Press, 2015). https://doi.org/10.17226/19007.

[2] Lacerenza, C. N., Marlow, S. L., Tannenbaum, S. I. \& Salas, E. Team development interventions: Evidence-based approaches for improving teamwork. Am. Psychol. 73, 517-531 (2018).

[3] See for example, Ambrose, S. A. et al. How Learning Works: Seven Research-Based Principles for Smart Teaching. (Jossey-Bass, 2010).

[4] Darling-Hammond, L., Wei, R. C., Andree, A., Richardson, N. \& Orphanos, S. Professional Learning in the Learning Profession: A Status Report on Teacher Development in the United States and Abroad. (National Staff Development Council, 2009).

[5] Guskey, T. R. \& Yoon, K. S. What Works in Professional Development? Phi Delta Kappan 90, 495-500 (2009).

[6] Gulamhussein, A. Teaching the Teachers: Effective Professional Development in an Era of High Stakes Accountability. (2013).

[7] Shore, L. M. et al. Inclusion and Diversity in Work Groups: A Review and Model for Future Research. J. Manag. 37, 1262-1289 (2011).

[8] Osland, J. S. \& Bird, A. Beyond Sophisticated Stereotyping: Cultural Sensemaking in Context [and Executive Commentaries]. Acad. Manag. Exec. 1993-2005 14, 65-79 (2000).

[9] Hunter, L. et al. Cultivating Scientist-and Engineer-Educators 2010: The Evolving Professional Development Program. in Learning from Inquiry in Practice ASPC 436, 3 (2010).

[10] St. John, M. \& Castori, P. The Design and Evolution of the Akamai Workforce Initiative. (2014).

[11] Goza, B. K. et al. Social Science at the Center for Adaptive Optics: Synergistic Systems of Program Evaluation, Applied Research, Educational Assessment, and Pedagogy. in Learning from Inquiry in Practice ASPC 436, 562581 (2010).

[12] Ball, T. \& Hunter, L. Developing and Sustaining a Science and Technology Center Education Program: "Inquiry" as a Means for Organizational Change and Institutional Legitimacy. in Learning from Inquiry in Practice ASPC 436, 469-489 (2010).

[13] Montgomery, R. et al. The Design and Implementation of the Akamai Maui Short Course. in Learning from Inquiry in Practice ASPC 436, 333-354 (2010).

[14] Seagroves, S. et al. Designers' Perspectives on Effective Professional Development for Scientist- and EngineerEducators. in Learning from Inquiry in Practice ASPC 436, 535-546 (2010). 
[15] Barnes, A. et al. Successfully Building a Diverse Telescope Workforce: The Design of the Akamai Internship Program in Hawai'i. in ASEE Annual Conference and Exposition (2018). https://peer.asee.org/31030

[16] Lin, Z. et al. Design of a pinhole mask unit for Keck NIRC2 AO instrument. in Proc. SPIE 11448-300 (2020).

[17] Ranka, T., Schöck, M., Chamarthi, S., Surya, A. \& Lu, J. Astrometric error analysis for ELTs: Analysis, methodology, and software tools. in Proc. SPIE 11450-67 (2020).

[18] Skidmore, W., International Science Development Teams, T. \& Science Advisory Committee, T. Thirty Meter Telescope Detailed Science Case: 2015. Res. Astron. Astrophys. 15, 1945-2140 (2015).

[19] N. Préel-Dumas \& W. Skidmore, private correspondence

[20] Williams, J. C., Phillips, K. W. \& Hall, E. V. Double Jeopardy? Gender Bias Against Women of Color in Science. UC Hastings College of the Law (2014) http://www.uchastings.edu/news/articles/2015/01/williams-doublejeopardy-report.php.

[21] Ebert-May, D. et al. What We Say Is Not What We Do: Effective Evaluation of Faculty Professional Development Programs. BioScience 61, 550-558 (2011).

\section{APPENDIX: LONG- AND MEDIUM-RANGE INDICATORS OF SUCCESS FOR TECI}

\section{Goal \#1: Advance early-career scientists' and engineers' involvement with TMT}

Long-range outcomes:

- TECI alums become members of TMT teams (instrument teams, instrument white paper teams, International Science Development Teams (ISDTs), etc.)

- TECI participants and alums are actively involved at TMT international community meetings

- TECI participants and alums author TMT-related publications, including TECI projects

- TECI participants and alums present TMT-related work at science or engineering conferences

- TECI alums advance their careers in astronomy, telescopes, or instrumentation

Medium-range indicators for the outcomes above:

- More participants pursue TECI projects after the Workshop

- TECI projects are recognized through TECI and TMT web materials

- TECI stimulates exchanges, visits, or hires across partner sites

Goal \#2: Contribute to making TMT teams more reflective of partner demographics Long-range outcomes:

- Balanced representation of TIO partners on ISDTs, instrument teams, and TMT Science Forum attendees

- Increase representation of women on ISDTs, instrument teams, TMT Science Forum attendees, and in team leadership roles

Medium-term indicators of the outcomes above:

- Balanced representation of TIO partners in leadership roles during TECI Workshop (instrument design team leads \& instrument design SAC)

- Increasing fraction of female participants at TECI Workshop, toward equal representation

- Gender equity in leadership roles during TECI Workshop and projects

- Balanced representation of TIO partners in TECI project continuation after the Workshop

- Gender equity in TECI project continuation after Workshop

Goal \#3: Support collaboration in the evolving TMT partnership

Long-range outcomes:

- TECI projects advance collaborations within TIO and produce required deliverables for TMT

- TECI projects advance collaborations within US-ELT partnership and produce required deliverables for USELT program

- TECI projects are widely used by TIO and US-ELT partnerships to stimulate collaboration

- Increase the number of multinational teams submitting science cases or use cases for TMT 
Medium-term indicators of the above outcomes include:

- International TECI project teams continue after workshop and build connections during in-person visit to a partner site

- TECI project teams develop preliminary products toward required deliverables for TMT and US-ELT

- $\quad$ TECI projects are increasingly aligned with TMT and US-ELT project needs 